STOCHASTIC MODELING AND CONTROL

BANACH CENTER PUBLICATIONS, VOLUME 122

INSTITUTE OF MATHEMATICS

POLISH ACADEMY OF SCIENCES

WARSZAWA 2020

\title{
ERGODIC IMPULSE CONTROL WITH CONSTRAINT: LOCALLY COMPACT CASE
}

\author{
JOSE LUIS MENALDI \\ Wayne State University, Department of Mathematics \\ Detroit, MI 48202, USA \\ E-mail: menaldi@wayne.edu \\ MAURICE ROBIN \\ Fondation Campus Paris-Saclay \\ 91190 Saint-Aubin, France \\ E-mail: maurice.robin@polytechnique.edu
}

\begin{abstract}
The impulse control problems for a Markov-Feller process with a long-term cost (ergodic) are considered, but the controls are allowed only when a signal arrives. This is referred to as control problems with constraint. Such problems are studied by the authors in SIAM J. Control. Optim. vol. 54, 55, 56 for the case of a compact metric state space and are extended in [Modeling, Stochastic Control, Optimization, and Applications, Springer, 2019, 427-450] to the situation of a locally compact state space with a uniform ergodicity assumption. The long term average cost problem is re-considered here with a non-uniform ergodicity assumption satisfied, for example, by a large class of diffusion processes in the whole space.
\end{abstract}

1. Introduction. A vast body of literature has been devoted to optimal stopping and impulse control of Markov processes, e.g., see the references in Bensoussan and Lions [2, 3], Bensoussan [1], Davis [4], and for ergodic impulse control, Palczewski and Stettner [21] and the references therein. A relatively small part of this literature concerns problems where constraints are imposed on the stopping times (see the references in [16]). In [13, 14, 15] we have studied optimal stopping and impulse control problems of a Markov

2010 Mathematics Subject Classification: Primary 49J40; Secondary 60J60, 60J75.

Key words and phrases: Markov-Feller processes, information constraints, impulse control, control by interventions, ergodic control.

The paper is in final form and no version of it will be published elsewhere. 
process $x_{t}$ when the stopping times must satisfy a constraint, namely, the control is allowed to take place only at the jump times of a given process $y_{t}$, these times representing the arrival of a signal. In these references, $x_{t}$ belongs to a compact metric space, and an extension to a locally compact Polish space appears in [16] under a uniform ergodicity assumption for what concerns the ergodic impulse control. In particular, this means that the case when $x_{t}$ is a diffusion process in the whole space $\mathbb{R}^{d}$ is not covered.

The aim of the present work is to address the ergodic impulse control problem with constraint, with a locally compact state space, under more general ergodicity assumptions. We use a similar method as in the previous work, namely, relying on an auxiliary problem in discrete time, which here turns out to give an HJB equation which is of the same type of the equation for semi-Markov decision processes (as in Jaśkiewicz [8], Luque-Vasquez and Hernandez-Lerma [11. We use specific additional assumptions, in particular to obtain an optimal control based on the exit times of a continuation region as it is usual for classical impulse control.

The paper is organized as follows. In Section 2, we introduce the statement of the problem (definitions the uncontrolled process, which is the two components process $\left(x_{t}, y_{t}\right)$, the admissible controls, the total average cost). Section 3 includes the main assumptions and preliminary properties. Section 4 presents the HJB equations. In Section 5, we study the existence of an optimal control based on the exit time of a continuation region. Section 6 gives comments on the ergodicity assumptions and Section 7 adds a few remarks on the case of diffusion processes.

2. Statement of the problem. In short, an impulse control problem for a MarkovFeller process with a long-term cost (ergodic) is considered, but the controls are allowed only when a signal arrives, but the details are many. Let us begin with some notation, definitions, comments, and the actual statement of our ergodic problem.

2.1. The uncontrolled process. First let us mention our Basic notation:

- $\mathbb{R}^{+}=[0, \infty[, E$ a locally compact, separable and complete metric space (in short, a locally compact Polish space), and also $\mathbb{N}_{0}=\{0,1, \ldots\}$ (i.e., natural numbers and 0 ), $\overline{\mathbb{N}}_{0}=\mathbb{N}_{0} \cup\{\infty\}$

- $\mathcal{B}(Z)$ the Borel $\sigma$-algebra of sets in $Z, B(Z)$ the space of real-valued Borel and bounded functions on $Z, C_{b}(Z)$ the space of real-valued continuous and bounded functions on $Z$, $C_{0}(Z)$ real-valued continuous functions vanishing at infinity on $Z$, i.e., a real-valued continuous function $v$ belongs to $C_{0}(Z)$ if and only if for every $\varepsilon>0$ there exists a compact set $K$ of $Z$ such that $|v(z)|<\varepsilon$ for every $z$ in $Z \backslash K{ }^{1}$ and also, if necessary, $B^{+}(Z), C_{b}^{+}(Z), C_{0}^{+}(Z)$ for nonnegative functions; usually either $Z=E$ or $Z=E \times \mathbb{R}^{+}$;

- the canonical space $D\left(\mathbb{R}^{+}, Z\right)$ of cad-lag functions, with its canonical process denoted by $z_{t}(\omega)=\omega(t)$ for any $\omega \in D\left(\mathbb{R}^{+}, Z\right)$, and its canonical filtration $\mathbb{F}^{0}=\left\{\mathcal{F}_{t}^{0}: t \geq 0\right\}$, $\mathcal{F}_{t}^{0}=\sigma\left(z_{s}: 0 \leq s \leq t\right)$.

\footnotetext{
${ }^{1}$ Typically $E=\mathbb{R}^{d}$ and this means that $v(z) \rightarrow 0$ as $|z| \rightarrow \infty$.
} 
Assumption 2.1. Let $\left(\Omega, \mathbb{F}, x_{t}, y_{t}, P_{x y}\right)$ be a (realization of a) strong and normal homogeneous Markov process, on $\Omega=D\left(\mathbb{R}^{+}, E \times \mathbb{R}^{+}\right)$with its canonical filtration universally completed $\mathbb{F}=\left\{\mathcal{F}_{t}: t \geq 0\right\}$ with $\mathcal{F}_{\infty}=\mathcal{F}$, where $\left(x_{t}, y_{t}\right)$ is the canonical process having values in $E \times \mathbb{R}^{+}$, and $\mathbb{E}_{x y}$ denotes the expectation relative to $P_{x y}$.

(a) It is also assumed that $x_{t}$ is a Markov process by itself (referred as the reduced state), with a $C_{0}$-semigroup $\Phi_{x}(t)$ (i.e., $\left.\Phi_{x}(t) C_{0}(E) \subset C_{0}(E), \forall t \geq 0\right)$, and infinitesimal generator $A_{x}$ with domain $\mathcal{D}\left(A_{x}\right) \subset C_{0}(E)$.

(b) The process $y_{t}$ (which is referred to as the signal process) has jumps to zero at times $\tau_{1}, \ldots, \tau_{n} \rightarrow \infty$ and $y_{t}=t-\tau_{n}$ for $\tau_{n} \leq t<\tau_{n+1}$ (i.e., $\tau_{1}$ is the time of the first jump -to zero- of $y_{t}$, each jump is 'the signal' and $y_{t}$ is exactly the 'time elapsed since the last jump or signal'), and if $y_{0}=0$ and $\tau_{0}=0$ then it is assumed that conditionally to $x_{t}$, the intervals between jumps $T_{n}=\tau_{n}-\tau_{n-1}$ are independent, identically distributed random variables with a continuous intensity function satisfying $k_{0} \leq \lambda(x, y) \leq k_{1}$, for suitable positive constants.

REMARK 2.1. Actually, we begin with a realization of the reduced state process $x_{t}$ on the canonical space $D\left(\mathbb{R}^{+}, E\right)$ and the signal process $y_{t}$ is constructed based on the given intensity $\lambda(x, y)$, and this procedure yields a $C_{0}\left(E \times \mathbb{R}^{+}\right)$-semigroup denoted by $\Phi_{x y}(t)$. Thus, in view of Palczewski and Stettner [19, all this implies that both semigroups $\Phi_{x}(t)$ and $\Phi_{x y}(t)$ have the Feller property, i.e., $\Phi_{x}(t) C_{b}(E) \subset C_{b}(E)$ and $\Phi_{x y}(t) C_{b}\left(E \times \mathbb{R}^{+}\right) \subset$ $C_{b}\left(E \times \mathbb{R}^{+}\right)$, and since only a strong and normal Markov process is assumed, the semigroup $\Phi_{x y}(t)$ is (initially) acting on $B\left(E \times \mathbb{R}^{+}\right)$and so, weak (or stochastic) continuity is deduced from the assumption of a cad-lag realization, which means that

$$
(x, y, t) \mapsto \mathbb{E}_{x y}\left\{h\left(x_{t}, y_{t}\right)\right\} \text { is a continuous function, }
$$

for any $h$ in $C_{b}\left(E \times \mathbb{R}^{+}\right)$. In [13, 14, 15] a probabilistic construction of the signal process $y_{t}$ was described, but there are other ways to construct $\Phi_{x y}(t)$. For instances, begin with the process $\left(x_{t}, \tilde{y}_{t}\right)$ with $\tilde{y}_{t}=y+t$ having infinitesimal generator $A^{0}=A_{x}+\partial_{y}$ and a $C_{0}\left(E \times \mathbb{R}^{+}\right)$-semigroup. Then, add the perturbation $B h(x, y)=\lambda(x, y)[h(x, 0)-h(x, y)]$, which is a bounded operator generating a $C_{0}\left(E \times \mathbb{R}^{+}\right)$-semigroup, with domain $\mathcal{D}(B)=$ $C_{0}\left(E \times \mathbb{R}^{+}\right)$. Hence $A_{x y}=A^{0}+B$ generates a $C_{0}\left(E \times \mathbb{R}^{+}\right)$-semigroup, with $\mathcal{D}\left(A_{x y}\right)=$ $\mathcal{D}\left(A^{0}\right)$, e.g., see Ethier and Kurtz [6, Section 1.7, pp. 37-40, Theorem 7.1]. Therefore $A_{x y}$ will also denote the weak infinitesimal generator in $C_{b}\left(E \times \mathbb{R}^{+}\right)$, in several places of the following sections.

REMARK 2.2. Note that Assumption 2.1(b) on $y_{t}$ means, in particular, that

$$
P_{x 0}\left\{T_{n} \in(t, t+d t) \mid x_{s}, 0 \leq s \leq t\right\}=\lambda\left(x_{t}, t\right) \exp \left(-\int_{0}^{t} \lambda\left(x_{s}, s\right) \mathrm{d} s\right) \mathrm{d} t,
$$

and then it is deduced that $\Phi_{x y}(t)$ has an infinitesimal generator $A_{x y}=A_{x}+A_{y}$ with

$$
A_{y} \varphi(x, y)=\partial_{y} \varphi(x, y)+\lambda(x, y)[\varphi(x, 0)-\varphi(x, y)]
$$

and recall that $\partial_{y}$ denotes the derivative with respect to $y$, and that $\lambda \geq 0$ and $\lambda \in$ $C_{b}\left(E \times \mathbb{R}^{+}\right)$. Moreover, using the law of $\tau_{1}$ as in (2) and the Feller property of $\left(x_{t}, y_{t}\right)$, it is also deduced that

$$
(x, y) \mapsto \mathbb{E}_{x y}\left\{g\left(x_{\tau_{1}}\right)\right\} \quad \text { belongs to } \quad C_{b}\left(E \times \mathbb{R}^{+}\right),
$$


for any $g$ in $C_{b}(E)$. Note that if we begin with a sequence $\left\{T_{1}, T_{2}, \ldots\right\}$ of IID random variables and $y_{0}=y$ then $\tau_{1}$ (the first signal) is random variable independent of $T_{1}, T_{2}, \ldots$ with distribution

$$
\left.\left.P_{x y}\left\{\tau_{1} \in\right] a, b\right]\right\}=\frac{\left.\left.P_{x 0}\left\{T_{1} \in\right] a+y, b+y\right]\right\}}{P_{x 0}\left\{T_{1} \geq y\right\}} .
$$

Furthermore, in turn, by applying Dynkin's formula to $A_{x y} \varphi(x, y)=f(x, y)$, it follows that

$$
(x, y) \mapsto \mathbb{E}_{x y}\left\{\int_{0}^{\tau_{1}} f\left(x_{t}, y_{t}\right) \mathrm{d} t\right\} \quad \text { is in } \quad C_{b}\left(E \times \mathbb{R}^{+}\right),
$$

for any $f$ in $C_{b}\left(E \times \mathbb{R}^{+}\right)$.

REMARK 2.3. Note that because $\lambda(x, y)$ is bounded (for $y$ near 0 is sufficient), there exists a constant $a$ such that $P_{x 0}\left\{\tau_{1} \geq a>0\right\} \geq a>0$, for any $x$ in $E$. Moreover, from Assumption 2.1(b) on the signal process $y_{t}$ we have

$$
\mathbb{E}_{x 0}\left\{\tau_{1}\right\}=\mathbb{E}_{x 0}\left\{\int_{0}^{\infty} t \lambda\left(x_{t}, t\right) \exp \left(-\int_{0}^{t} \lambda\left(x_{s}, s\right) \mathrm{d} s\right) \mathrm{d} t\right\},
$$

so if $\lambda(x, y) \leq k_{1}<\infty$, for every $y \geq 0$, and $x \in E$, then $\mathbb{E}_{x 0}\left\{\tau_{1}\right\} \geq a_{1}=1 / k_{1}$. Also, the condition $\mathbb{E}_{x 0}\left\{\tau_{1}\right\} \leq a_{2}$ is satisfied since by Assumption 2.1 (b) $\lambda(x, y) \geq k_{0}>0$ for $y \geq y_{0}, x \in E$, then $a_{2}=y_{0}+1 / k_{0}$. Moreover, since $\lambda(x, y)$ is a continuous function in $E \times \mathbb{R}^{+}$, the continuity of $E_{x y}\left\{\tau_{1}\right\}$ follows.

DeFinition 2.1 (with comments). The expression

$$
\left\{X_{n}=x_{\tau_{n}}, n=0,1, \ldots\right\},
$$

with $\tau_{0}=0$ and $X_{0}=x$, defines a homogeneous Markov chain in $E$ with respect to the filtration $\mathbb{G}=\left\{\mathcal{G}_{n}: n=0,1, \ldots\right\}$ obtained from $\mathbb{F}$, namely, $\mathcal{G}_{n}=\mathcal{F}_{\tau_{n}}$. In this context, if

$$
\tau=\inf \left\{t>0: y_{t}=0\right\},
$$

is considered as a functional on $\Omega$, then the sequence of signals (i.e., the instants of jumps for $y_{t}$ ) is defined by recurrence

$$
\tau_{k+1}=\inf \left\{t>\tau_{k}: y_{t}=0\right\}, \quad \forall k=1,2, \ldots,
$$

with $\tau_{1}=\tau$, and by convenience, set $\tau_{0}=0$. Let us also mention that Remark 2.3 yields: there exists a constant $a_{1}$ such that

$$
P_{x 0}\left\{\tau \geq a_{1}>0\right\} \geq a_{1}>0, \quad \forall x \in E,
$$

and by Assumption 2.1. there exists another constant $a_{2}>0$ such that

$$
\mathbb{E}_{x 0}\{\tau\} \leq a_{2}, \quad \forall x \in E,
$$

therefore,

$$
0<a_{1} \leq \tau(x):=\mathbb{E}_{x 0}\{\tau\} \leq a_{2}, \quad \forall x \in E,
$$

for some real numbers $a_{1}, a_{2}$. 
2.2. The controlled process. For a detailed construction we refer to Bensoussan and Lions 3] (see also Davis [4, Lepeltier and Marchal [10], Robin [22, Stettner [24]).

Let us consider $\Omega^{\infty}=\left[D\left(\mathbb{R}^{+} ; E \times \mathbb{R}^{+}\right)\right]^{\infty}$, and define $\mathcal{F}_{t}^{0}=\mathcal{F}_{t}$ and $\mathcal{F}_{t}^{n+1}=\mathcal{F}_{t}^{n} \otimes \mathcal{F}_{t}$, for $n \geq 0$, where $\mathcal{F}_{t}$ is the universal completion of the canonical filtration as previously.

An arbitrary impulse control $\nu$ (not necessarily admissible at this stage) is a sequence $\left(\theta_{n}, \xi_{n}\right)_{n \geq 1}$, where $\theta_{n}$ is a stopping time of $\mathcal{F}_{t}^{n-1}, \theta_{n} \geq \theta_{n-1}$, and the impulse $\xi_{n}$ is $\mathcal{F}_{\theta_{n}}^{n-1}$ measurable random variable with values in $E$.

The coordinate in $\Omega^{\infty}$ has the form $\left(x_{t}^{0}, y_{t}^{0}, x_{t}^{1}, y_{t}^{1}, \ldots, x_{t}^{n}, y_{t}^{n}, \ldots\right)$, and for any impulse control $\nu$ there exists a probability $P_{x y}^{\nu}$ on $\Omega^{\infty}$ such that the evolution of the controlled process $\left(x_{t}^{\nu}, y_{t}^{\nu}\right)$ is given by the coordinates $\left(x_{t}^{n}, y_{t}^{n}\right)$ of $\Omega^{\infty}$ when $\theta_{n} \leq t<\theta_{n+1}, n \geq 0$ (setting $\left.\theta_{0}=0\right)$, i.e., $\left(x_{t}^{\nu}, y_{t}^{\nu}\right)=\left(x_{t}^{n}, y_{t}^{n}\right)$ for $\theta_{n} \leq t<\theta_{n+1}$. Note that clearly $\left(x_{t}^{\nu}, y_{t}^{\nu}\right)$ is defined for any $t \geq 0$, but $\left(x_{t}^{i}, y_{t}^{i}\right)$ is only used for any $t \geq \theta_{i}$, and $\left(x_{\theta_{i}}^{i-1}, y_{\theta_{i}}^{i-1}\right)$ is the state at time $\theta_{i}$ just before the impulse (or jump) to $\left(\xi_{i}, y_{\theta_{i}}^{i-1}\right)=\left(x_{\theta_{i}}^{i}, y_{\theta_{i}}^{i}\right)$, as long as $\theta_{i}<\infty$. Remark that the impulse control $\nu=\left\{\left(\theta_{i}, \xi_{i}\right): i \geq 1\right\}$ and the probability $P_{x y}^{\nu}$ are constructed by means of a sequential (or inductive) procedure, and it may be convenient to add $\theta_{0}=0$ and $\xi_{0}=x$, which is not considered as an impulse. Hence, $\left\{\left(x_{t}^{0}, y_{t}^{0}\right): t \geq 0\right\}$ is the uncontrolled Markov evolution (of the state) and $\left\{\left(x_{t}^{i}, y_{t}^{i}\right): t \geq \theta_{i}\right\}$ denotes the Markov evolution after the $i$-impulse, i.e., only the first $i$ impulses are applied and the Markov process restart anew at time $\theta_{i}<\infty$ with initial condition $\left(x_{\theta_{i}}^{i}, y_{\theta_{i}}^{i}\right)=\left(\xi_{i}, 0\right)$, since $y_{\theta_{i}}^{i-1}=0$. Also the sequence $\left\{\tau_{k}^{i}: k \geq 1\right\}$ of signals after $\theta_{i}$ is given by the functional $\tau_{k+1}^{i}=\inf \left\{t>\tau_{k}^{i}: y_{t}^{i}=0\right\}$, beginning with $\tau_{0}^{i}=\theta_{i}<\infty$, and using the convention $\inf \{\emptyset\}=\infty$. For the sake of simplicity, we will not always indicate, in the sequel, the dependency of $\left(x_{t}^{\nu}, y_{t}^{\nu}\right)$ with respect to $\nu$. A Markov impulse control $\nu$ is identified by a closed subset $S$ of $E \times \mathbb{R}^{+}$and a Borel measurable function $(x, y) \mapsto \xi(x, y)$ from $S$ into $C=E \times \mathbb{R}^{+} \backslash S$, with the following meaning: intervene only when the the process $\left(x_{t}, y_{t}\right)$ is leaving the continuation region $C$ and then apply an impulse $\xi(x, y)$, while in the stopping region $S$, moving back the process to the continuation region $C$, i.e., $\theta_{i+1}=\inf \left\{t>\theta_{i}:\left(x_{t}^{i}, y_{t}^{i}\right) \in S\right\}$, with the convention that $\inf \{\emptyset\}=\infty$, and $\xi_{i+1}=$ $\xi\left(x_{\theta_{i+1}}^{i}, y_{\theta_{i+1}}^{i}\right)$, for any $i \geq 0$, as long as $\theta_{i}<\infty$.

Now, recalling that $\tau_{n}$ are the arrival times of the signal given by (8), the admissible controls are defined as follows:

\section{DEFINITION 2.2.}

(i) A stopping time $\theta$ is called admissible if almost surely there exists $n=\eta(\omega) \geq 1$ such that $\theta(\omega)=\tau_{\eta(\omega)}(\omega)$, or equivalently if $\theta$ satisfies $\theta>0$ and $y_{\theta}=0$ a.s. If $\theta=0$ (i.e., $\eta=0$ ) is allowed, then $\theta$ is called a zero-admissible stopping time.

(ii) An impulse control $\nu=\left\{\left(\theta_{i}, \xi_{i}\right), i \geq 1\right\}$ as above is called admissible, if each $\theta_{i}$ is admissible (i.e., $\theta_{i}>0$ and $y_{\theta_{i}}=0$ ), and $\xi_{i} \in \Gamma\left(x_{\theta_{i}}^{i-1}\right)$. The set of admissible impulse controls is denoted by $\mathcal{V}$.

(iii) If $\theta_{1}=0$ is allowed, then $\nu$ is called zero-admissible. The set of zero-admissible impulse controls is denoted by $\mathcal{V}_{0}$.

(iv) An admissible Markov impulse control corresponds to a stopping region $S=$ $S_{0} \times\{0\}$ with $S_{0} \subset E$, and an impulse function satisfying $\xi(x, 0)=\xi_{0}(x) \in \Gamma(x)$, for any $x \in S_{0}$, and therefore, if $\left\{\left(x_{t}^{0}, y_{t}^{0}\right): t \geq 0\right\}$ is the uncontrolled Markov evolution (of 
the state) and $\left\{\left(x_{t}^{i}, y_{t}^{i}\right): t \geq \theta_{i}\right\}$ denotes the Markov evolution after the $i$-impulse then $\eta_{0}=0, \tau_{0}^{0}=0, \theta_{0}=\tau_{0}^{0}, \xi_{0}=x, \tau_{k}^{0}=\inf \left\{t>\tau_{k-1}^{0}: y_{t}^{0}=0\right\}(\forall k \geq 1), \eta_{1}=\inf \left\{k>\eta_{0}:\right.$ $\left.x_{\tau_{k}^{0}}^{0} \in S_{0}\right\}, \theta_{1}=\tau_{\eta_{1}}^{0}, \tau_{\eta_{1}}^{1}=\theta_{1}, \xi_{1}=\xi\left(x_{\theta_{1}}^{0}, 0\right)$, and next, $\tau_{k}^{1}=\inf \left\{t>\tau_{k-1}^{1}: y_{t}^{1}=0\right\}$ $\left(\forall k>\eta_{1}\right), \eta_{2}=\inf \left\{k>\eta_{1}: x_{\tau_{k}^{1}}^{1} \in S_{0}\right\}, \theta_{2}=\tau_{\eta_{2}}^{1}, \tau_{\eta_{2}}^{2}=\theta_{2}, \xi_{2}=\xi\left(x_{\theta_{2}}^{1}, 0\right)$, and so forth. For a zero-admissible Markov impulse control, it suffices to use $\eta_{1}=\inf \left\{k \geq \eta_{0}\right.$ : $\left.x_{\tau_{k}^{0}}^{0} \in S_{0}\right\}$, i.e., to replace $k>\eta_{0}$ with $k \geq \eta_{0}$, within the construction of $\eta_{1}$ in the previous iteration.

As seen later, it will be useful to consider an auxiliary problem in discrete time, for the Markov chain $X_{n}=x_{\tau_{n}}$, with the filtration $\mathbb{G}=\left\{\mathcal{G}_{n}, n \geq 0\right\}, \mathcal{G}_{n}=\mathcal{F}_{\tau_{n}}^{n-1}$. The impulses occur at the stopping times $\eta_{i}$ with values in the set $\mathbb{N}=\{0,1,2, \ldots\}$ and are related to $\theta_{i}$ by $\eta_{i}=\inf \left\{k>\eta_{i-1}: \theta_{i}=\tau_{k}^{i}\right\}$ for admissible controls $\left\{\theta_{i}\right\}$ and similarly for zero-admissible controls with $\eta_{i}=\inf \left\{k \geq \eta_{i-1}: \theta_{i}=\tau_{k}^{i}\right\}$. The discrete time impulse control problem has been consider in Bensoussan [1], Stettner [23]. Thus

Definition 2.3. If $\nu=\left\{\left(\eta_{i}, \xi_{i}\right), i \geq 1\right\}$ is a sequence of $\mathbb{G}$-stopping times and $\mathcal{G}_{\eta_{i}}$-measurable random variables $\xi_{i}$, with $\xi_{i} \in \Gamma\left(x_{\tau_{\eta_{i}}}\right), \eta_{i}$ increasing and $\eta_{i} \rightarrow+\infty$ a.s., then $\nu$ is referred to as an admissible discrete time impulse control if $\eta_{1} \geq 1$. If $\eta_{i} \geq 0$ is allowed, it is referred as an zero-admissible discrete time impulse control.

For an admissible impulse control $\nu$, with a running cost $f(x, y)$ and a cost-per-impulse $c(x, \xi)$, the average cost is defined as

$$
\begin{aligned}
J^{T}(0, x, y, \nu) & =\mathbb{E}_{x y}^{\nu}\left\{\int_{0}^{T} f\left(x_{s}^{\nu}, y_{s}^{\nu}\right) \mathrm{d} s+\sum_{i} \mathbb{1}_{\theta_{i} \leq T} c\left(x_{\theta_{i}}^{i-1}, \xi_{i}\right)\right\}, \\
J(x, y, \nu) & =\liminf _{T \rightarrow \infty} \frac{1}{T} J^{T}(0, x, y, \nu) .
\end{aligned}
$$

The ergodic control problem is to characterize

$$
\mu(x, y)=\inf _{\nu \in \mathcal{V}} J(x, y, \nu),
$$

and to find an optimal control. Also, consider an auxiliary problem given as

$$
\begin{aligned}
\mu_{0}(x, y) & =\inf _{\nu \in \mathcal{V}_{0}} \tilde{J}(x, y, \nu), \quad \text { with } \\
\tilde{J}(x, y, \nu) & =\liminf _{n \rightarrow \infty} \frac{1}{\mathbb{E}_{x y}^{\nu}\left\{\tau_{n}\right\}} J^{\tau_{n}}(0, x, y, \nu),
\end{aligned}
$$

and $J^{\tau_{n}}(0, x, y, \nu)$ as in 12 with $T=\tau_{n}$. Later it is shown that $\mu(x, y)=\mu_{0}(x, y)$ is a constant.

REMARK 2.4. Similarly to [15, Remark 5.4]), it can be shown that the results which follow are the same if 'lim inf' is replaced by 'lim sup' in the definition of the cost either 12 or 14 .

3. Main assumptions and preliminaries. It is assumed that the running cost $f(x, y)$ and the cost-per-impulse $c(x, \xi)$ satisfy

$$
\begin{aligned}
& f: E \times \mathbb{R}^{+} \rightarrow \mathbb{R}^{+}, \text {bounded and continuous, } \\
& c: E \times E \rightarrow\left[c_{0},+\infty\left[, c_{0}>0,\right. \text { bounded and continuous, }\right.
\end{aligned}
$$


Moreover, for any $x \in E$, the possible impulses must be in $\Gamma(x)=\{\xi \in E:(x, \xi) \in \Gamma\}$, where $\Gamma$ is a given analytic set in $E \times E$. Actually, for every $x$ in $E$ the following properties are assumed to hold

$$
\begin{aligned}
& \emptyset \neq \Gamma(x) \text { is compact, } \quad \forall \xi \in \Gamma(x), \Gamma(\xi) \subset \Gamma(x), \\
& c(x, \xi)+c\left(\xi, \xi^{\prime}\right) \geq c\left(x, \xi^{\prime}\right), \quad \forall \xi \in \Gamma(x), \forall \xi^{\prime} \in \Gamma(\xi) \subset \Gamma(x) .
\end{aligned}
$$

Finally, by defining the operator $M$

$$
M v(x)=\inf _{\xi \in \Gamma(x)}\{c(x, \xi)+v(\xi)\}
$$

it is assumed that

$M$ maps $C_{b}(E)$ into $C_{b}(E)$, and there exists a measurable selector

$$
\hat{\xi}(x)=\hat{\xi}(x, v) \text { realizing the infimum in } M v(x), \forall x, v .
$$

REMARK 3.1 .

(a) The last condition in 16 is to ensure that simultaneous impulses are never optimal.

(b) 18 requires some regularity property of $\Gamma(x)$, e.g., see Davis [4].

(c) It is possible (but not necessary) that $x$ belongs to $\Gamma(x)$, actually, even $\Gamma(x)=E$ whenever $E$ is compact, satisfies the assumptions. However, an impulse occurs when the system moves from a state $x$ to another state $\xi \neq x$, i.e., it suffices to avoid (or not to allow) impulses that moves $x$ to itself, since they have a higher cost.

The transition probability of the Markov chain $X_{n}$ as in Definition 2.1 is $P(x, B)=$ $\mathbb{E}_{x 0} \mathbb{1}_{B}\left(x_{\tau}\right)$, with $\tau$ defined by (7), for any Borel subset $B \subset E$. Also, define the operator

$$
P v(x)=\mathbb{E}_{x 0}\left\{v\left(x_{\tau}\right)\right\}, \quad \forall x \in E,
$$

for every bounded and measurable function $v$, i.e., $P \mathbb{1}_{B}(x)=P(x, B)$, for every $x \in E$ and $B \in \mathcal{B}(E)$, the Borel $\sigma$-algebra on $E$.

We assume the following conditions:

$$
\text { there exists a continuous function } V: E \rightarrow[1, \infty[
$$

and there exist a closed set $C$ and an open set $D$ in $E$ such that $C \subset D$, a probability $m$ on $E$ satisfying

$$
\begin{aligned}
& 0<m(C)<1=m(D) \text { and } \sup \{V(x): x \in C\}<\infty, \\
& P V(x) \leq \beta_{1} V(x)+\beta_{2} \mathbb{1}_{C}(x), \quad \forall x \in E, \\
& P(x, B) \geq \beta_{0} m(B), \quad \forall x \in D, \forall B \subset D, B \in \mathcal{B}(E),
\end{aligned}
$$

for a suitable constants $\beta_{0}, \beta_{1}$ in $] 0,1\left[\right.$, and $\beta_{2}>0$. As shown in the next section, the function $P V$ is necessarily continuous.

Let us now give some preliminary properties.

Lemma 3.1. The operator $P$ defined by 19 maps $C_{b}(E)$ into itself.

Proof. In view of the equality

$$
P v(x)=\mathbb{E}_{x}\left\{\int_{0}^{\infty} \lambda\left(x_{t}, t\right) \exp \left(-\int_{0}^{t} \lambda\left(x_{s}, s\right) \mathrm{d} s\right) v\left(x_{t}\right) \mathrm{d} t\right\},
$$


for any $g$ in $C_{b}\left(E \times \mathbb{R}^{+}\right)$, define the semigroup

$$
\tilde{\Phi}(t) g(x, y)=\mathbb{E}_{x}\left\{\exp \left(-\int_{0}^{t} \lambda\left(x_{s}, y+s\right) \mathrm{d} s\right) g\left(x_{t}, y+t\right)\right\},
$$

which satisfies

$$
P v(x)=\int_{0}^{\infty} \tilde{\Phi}(t) g(x, 0) \mathrm{d} t, \quad \text { with } g(x, y)=\lambda(x, y) v(x) .
$$

Since $\lambda(x, y) \geq k_{0}$, the bound $\|\tilde{\Phi}(t) g(x, 0)\| \leq \mathrm{e}^{-k_{0} t}\|g\|$ enables the use of Lebesgue Theorem to show that if $\{\tilde{\Phi}(t): t \geq 0\}$ is Feller then $P v(x)$ is continuous, and moreover, since also $\lambda$ is bounded, the operator $P$ maps $C_{b}(E)$ into itself.

Therefore, it suffices to prove that $\{\tilde{\Phi}(t): t \geq 0\}$ is a $C_{0}$-semigroup. To this purpose, note that by assumption, $\Phi^{0}(t) g(x, y)=\mathbb{E}_{x}\left\{g\left(x_{t}, y+t\right)\right\}$ is a $C_{0}$-semigroup with infinitesimal generator $A^{0}=A_{x}+\partial_{y}$. Accordingly to Dynkin [5, Theorem 9.7, pp. 298-299], the infinitesimal generator of $\{\tilde{\Phi}(t): t \geq 0\}$ is $\tilde{A}=A^{0}-\lambda I$ and $\mathcal{D}(\tilde{A})=\mathcal{D}\left(A^{0}\right)$. Thus, $\{\tilde{\Phi}(t): t \geq 0\}$ is a $C_{0}$-semigroup as a consequence of Dynkin [5, Theorems 2.9 and 2.10, pp. 74-77]. Note that there is no need of $\tilde{\Phi}(t)$ when $\lambda(x, y)=\lambda(y)$.

Let us comment on condition 20 .

Lemma 3.2. Under Assumption 2.1 and (20), the function PV is continuous.

Proof. Since $V$ is a continuous and positive function (a priori unbounded) there exists an increasing sequence $\left\{V_{k}\right\} \subset C_{b}(E)$ such that $V_{k}(x) \uparrow V(x)$ for every $x \in E$, e.g., $V_{k}(x)=\min \{V(x), k\}, k=1,2, \ldots$ Also note that if $v-u \geq 0$ are measurable functions then $P v-P u \geq 0$. Hence, the monotone convergence ensures that $P V_{k}(x) \uparrow P V(x)$ and Dini's Theorem implies that $P V_{k} \rightarrow P V$ uniformly on compact sets of $E$. Therefore, if $x_{n} \rightarrow x$ then $P V_{k}\left(x_{n}\right) \uparrow P V\left(x_{n}\right)$ uniformly in $n$. Also, because $V_{k}$ belongs to $C_{b}(E)$ it follows, that for every $k$ fixed, $P V_{k}\left(x_{n}\right) \rightarrow P V_{k}(x)$ as $n \rightarrow \infty$. Hence, the inequality

$$
\begin{aligned}
\left|P V\left(x_{n}\right)-P V(x)\right| \leq\left|P V\left(x_{n}\right)-P V_{k}\left(x_{n}\right)\right|+\left|P V_{k}\left(x_{n}\right)-P V_{k}(x)\right| & \\
& +\left|P V_{k}(x)-P V(x)\right|, \quad \forall n, k,
\end{aligned}
$$

shows that $P V\left(x_{n}\right) \rightarrow P V(x)$ as desired.

For the reasons which will appear later, we introduce a function $W=W(x)$ to replace $V(x)$. The following Lemma is shown in Jaśkiewicz [8, Lemma 3.1, and pp. 2572-73].

LEMma 3.3. Under the ergodic assumption (20) and 21) and with the same $\beta_{0}, \beta_{1}, \beta_{2}$, $C$ and $D$, the function $W(x)=V(x)+\beta_{2} / \beta_{0}$ satisfies

$$
\begin{aligned}
& P W(x) \leq \beta^{\prime} W(x)+\mathbb{1}_{C}(x) \beta_{2}, \quad \forall x \in E, \\
& P W(x) \leq \beta^{\prime} W(x)+\beta_{0} \gamma(x) \int_{D} W(z) m(\mathrm{~d} z), \quad \forall x \in E, \\
& P(x, B) \geq \beta_{0} \gamma(x) m(B), \quad \forall x \in D, \forall B \subset D, \quad B \in \mathcal{B}(E),
\end{aligned}
$$

where $\gamma$ is a continuous functions on $E$ such that $0 \leq \gamma \leq 1, \gamma=1$ in $C, \gamma=0$ on $E \backslash D$, and $\beta^{\prime}=\left(\beta_{0} \beta_{1}+\beta_{2}\right) /\left(\beta_{0}+\beta_{2}\right)$ is a constant in $] 0,1[$. 
In is convenient to denote by $B_{W}(E)$ [and $C_{W}(E)$ ] the space of real-valued measurable [continuous] functions with finite $W$-weighted norm

$$
\|v\|_{W}=\sup \{|v(x)| / W(x): x \in E\} .
$$

Lemma 3.4. The operator $P$ defined by 19 maps $C_{W}(E)$ into itself.

Proof. One checks that

$$
|P v(x)| \leq\|v(x)\|_{W} P W(x), \quad \forall v \in B_{W}(E),
$$

and the inequality $P W(x) \leq \beta^{\prime} W(x)+k$ (Lemma 3.3) yields

$$
\|P v(x)\|_{W} \leq k^{\prime}\|v(x)\|_{W}, \quad \forall v \in B_{W}(E),
$$

and some constant $k^{\prime}>0$.

To prove that $P v$ is continuous for any $v$ in $C_{W}(E)$, consider the continuous and positive functions $v^{ \pm}=\|v(x)\|_{W} W \pm v$. Since the space $E$ is $\sigma$-compact (i.e., $E$ is the union of a sequence of compacts), there exists an increasing sequence $\left\{v_{k}^{ \pm}: k \geq 1\right\}$ in $C_{b}(E)$ such that $v_{k}^{ \pm}(x) \uparrow v^{ \pm}(x)$ for every $x$ in $E$. Now, if $x_{n} \rightarrow x$ then, as $n \rightarrow \infty$,

$$
P v_{k}^{ \pm}(x)=\liminf _{n} P v_{k}^{ \pm}\left(x_{n}\right) \leq \liminf _{n} P v^{ \pm}\left(x_{n}\right)
$$

and as $k \rightarrow \infty$, we deduce that $x \mapsto P v^{ \pm}(x)$ is lower semi-continuous. Because $x \mapsto$ $P W(x)$ is continuous, this implies that $x \mapsto \pm P v(x)$ is also lower semi-continuous, which means that $x \mapsto P v(x)$ is continuous.

In the following sections, we need to assume that

$$
M \text { maps } C_{W}(E) \text { into itself. }
$$

A simple situation is

LEMmA 3.5. If $\Gamma(x) \subset K_{0}$, a fixed compact set in $E$, for any $x$ in $E$; then $M v$ is bounded, for any real-valued continuous function $v$ on $E$, and therefore, $\|M v\|_{W}(E)<\infty$.

Proof. Indeed, from the inequality

$$
|M v(x)| \leq \sup \{c(x, \xi): \xi \in \Gamma(x), x \in E\}+\sup \left\{|v(\xi)|: \xi \in K_{0}\right\},
$$

the result follows.

LEMMA 3.6. If the operator $M$ maps $C_{b}(E)$ into itself, $\Gamma(x)$ is pre-compact for every $x$ in $E$, the multivalued-function $x \mapsto \Gamma(x)$ is continuous in the Hausdorff metric of sets, and that the Polish space $E$ is locally compact, then $v$ continuous implies Mv continuous.

Proof. Indeed, if d denotes the metric on $E$ then, for every $\varepsilon>0$ the set $K_{\varepsilon}(x)=\left\{\xi^{\prime}\right.$ : $\left.\mathrm{d}\left(\xi, \xi^{\prime}\right) \leq \varepsilon, \xi \in \bar{\Gamma}(x)\right\}$ is a compact set. The convergence in the Hausdorff metric implies that given any $\varepsilon>0$ there exists $r>0$ such that $\mathrm{d}\left(x, x^{\prime}\right)<r$ implies $\mathrm{d}_{H}\left(\Gamma(x), \Gamma\left(x^{\prime}\right)\right)<\varepsilon$, and therefore $\Gamma\left(x^{\prime}\right) \subset K_{\varepsilon}(x)$, for any $x^{\prime}$ satisfying $\mathrm{d}\left(x, x^{\prime}\right)<r$. Thus, if $v=\tilde{v}$ on $K_{\varepsilon}(x)$ then $M v\left(x^{\prime}\right)=M \tilde{v}\left(x^{\prime}\right)$, for any $x^{\prime}$ in $B_{r}(x)=\left\{x^{\prime}: \mathrm{d}\left(x, x^{\prime}\right) \leq r\right\}$. Since continuous functions are bounded on compact sets, there exists $v_{b}$ in $C_{b}(E)$ such that $v=v_{b}$ on $K_{\varepsilon}(x)$. Hence, because $M v\left(x^{\prime}\right)=M v_{b}\left(x^{\prime}\right)$ for every $x^{\prime}$ in $B_{r}$ and $M v_{b}$ belongs to $C_{b}(E)$, we deduce that $M v$ is continuous on $B_{r}$, i.e., the operator $M$ maps continuous functions into continuous functions, as desired. 
LEMMA 3.7. If

$$
\exists K>0 \text { such that } \sup _{\xi \in \Gamma(x)} W(\xi) \leq K W(x), \quad \forall x \in E,
$$

then the operator $M$ satisfies

$$
\|M v\|_{W} \leq K^{\prime}\left(1+\|v\|_{W}\right), \quad v \in B_{W}(E),
$$

for some constant $K^{\prime}>0$.

Proof. From $|v(x)| \leq\|v\|_{W} W(x)$ follows

$$
M v(x) \geq \inf _{\xi \in \Gamma(x)} v(\xi) \geq-\|v\|_{W} \sup _{\xi \in \Gamma(x)} W(\xi) \geq-\|v\|_{W} K W(x)
$$

and

$$
M v(x) \leq K_{c}+\|v\|_{W} \inf _{\xi \in \Gamma(x)} W(\xi) \leq K_{c}+\|v\|_{W} K W(x),
$$

with $K_{c}=\sup \{|c(x, \xi)|: \xi \in \Gamma(x), x \in E\}$. Hence the desired estimate holds with $K^{\prime}=\max \left\{K_{c}, K\right\}$.

REMARK 3.2. Note that if $W(x)$ has at most a polynomial growth with $E=\mathbb{R}^{d}$ and $\Gamma(x)-x$ is contained in a fixed bounded set, then the assumption of Lemma 3.7 is true. However, the assumption of Lemma 3.7 may hold even when $\Gamma(x)-x$ is not bounded.

4. HJB equation. The Dynamic Programming Principle shows (heuristically, see [15], Section 3] that, with $w_{0}(x)=w_{0}(x, 0)$, and for every $x$ in $E$,

$$
w_{0}(x)=\min \left\{\mathbb{E}_{x 0}\left\{\int_{0}^{\tau}\left[f\left(x_{t}, y_{t}\right)-\mu_{0}\right] \mathrm{d} t+w_{0}\left(x_{\tau}\right)\right\}, M w_{0}(x)\right\},
$$

is the corresponding Hamilton-Jacobi-Bellman (HJB) equations in a weak form with two unknowns $\mu_{0}$ and $w_{0}$. Also, both problems are related (logically) by the condition

$$
\begin{aligned}
& w(x, y)=\mathbb{E}_{x y}\left\{\int_{0}^{\tau}\left[f\left(x_{t}, y_{t}\right)-\mu_{0}\right] \mathrm{d} t+w_{0}\left(x_{\tau}\right)\right\}, \quad \forall x \in E, y \geq 0, \\
& w(x, y)=w_{0}(x, y), \quad \forall x \in E, y>0,
\end{aligned}
$$

and so, if $w_{0}(x)$ is known then the last two equality yield $w(x, y)$ and $w_{0}(x, y)$. Recall that $\tau$ is defined by (7) and that since $w(x, y)=w_{0}(x, y)$ for any $x \in E$ and $y>0$, it may be convenient to write $w_{0}(x)=w_{0}(x, 0)$ as long as no confusion arises. Note that the functions $w(x, y)$ and $w_{0}(x)$ may be called potentials, and a priori, they are not costs, but they are used to determine an optimal control.

REMARK 4.1. Note that conditions (24) do not look like a HJB equation, however, we will check later the relation $w_{0}(x)=\min \{w(x, 0), M w(x, 0)\}$, and so, conditions (24) are indeed equivalent to the HJB equation

$$
w(x, y)=\mathbb{E}_{x 0}\left\{\int_{0}^{\tau}\left[f\left(x_{t}, y_{t}\right)-\mu_{0}\right] \mathrm{d} t+\min \{w(x, 0), M w(x, 0)\}\right\},
$$

for $\left(w, \mu_{0}\right)$.

Let us remark that the HJB equation 23 is equivalent to

$$
w_{0}(x)=\min \left\{M w_{0}(x), \ell(x)-\mu_{0} \tau(x)+P w_{0}(x)\right\},
$$


where

$$
\ell(x)=\mathbb{E}_{x 0}\left\{\int_{0}^{\tau} f\left(x_{s}, y_{s}\right) \mathrm{d} s\right\}, \quad \tau(x)=\mathbb{E}_{x 0}\{\tau\},
$$

with $\tau$ as in (7), and in view of Assumption 2.1(b) (see also Remark 2.2), the operator

$$
\operatorname{Ph}(x)=\mathbb{E}_{x 0}\left\{h\left(x_{\tau}\right)\right\}
$$

has been defined in 19 , initially from $C_{b}(E)$ into itself, but Lemma 3.4 shows that also it maps $C_{W}(E)$ into itself. Note that 10 yields

$$
0 \leq \ell(x) \leq a_{2}\|f\|
$$

Moreover, from the Feller property of $x_{t}$ and the law of $\tau$ it follows that $\ell(x)$ is continuous.

THEOREM 4.1. Under Assumption 2.1 and (15), 16), and (18) which is complemented with (20), 21) and 22, there exists a solution $\left(\mu_{0}, w_{0}\right)$ in $\mathbb{R}^{+} \times C_{W}(E)$ of 25], and therefore, of 23 .

Proof. As in [15, the HJB 25 can be written as

$$
w_{0}(x)=\inf _{\xi \in \Gamma(x) \cup\{x\}}\left\{L(x, \xi)-\mu_{0} \tau(\xi)+P w_{0}(\xi)\right\}
$$

with $L(x, \xi)=\ell(\xi)+\mathbb{1}_{\xi \neq x} c(x, \xi)$, which is a particular case of the average cost optimality equation studied in Jaśkiewicz [8] (among others). In our case, however, the function $L$ and the set-function $x \mapsto \Gamma(x) \cup\{x\}$ are not continuous, so that a slight adaptation is necessary as follows. Indeed, define $P^{\prime}(\cdot \mid x)=P(\cdot \mid x)-\beta_{0} \gamma(x) m(\cdot)$ and consider the operator

$$
T v(x)=\inf \left\{L(x, \xi)-g \tau(\xi)+P^{\prime} v(\xi): \xi \in \Gamma(x) \cup\{x\}\right\},
$$

where the constant $g$ is given by

$$
g=\inf _{\nu \in \mathcal{S}}\left\{\liminf _{n \rightarrow \infty} \frac{1}{\mathbb{E}_{x 0}^{\nu}\left\{\tau_{n}\right\}} \mathbb{E}_{x 0}^{\nu}\left\{\sum_{k=0}^{n-1} L\left(X_{k}, \xi_{k}\right)\right\}\right\}
$$

and $\mathcal{S}$ is the set of all stationary policies. Now, due to the continuity of $\gamma$, the function $P^{\prime} v(x)$ is continuous if $v$ is so. Even if not all assumptions in Jaśkiewicz [8] are satisfied in our case, the alternative expression of $T$ as

$$
T v(x)=\min \left\{\inf _{\xi \in \Gamma(x)}\left\{\ell(\xi)+c(x, \xi)-g \tau(\xi)+P^{\prime} v(\xi)\right\}, \ell(x)-g \tau(x)+P^{\prime} v(x)\right\},
$$

shows, after using assumption 22 , that $T$ maps $C_{W}(E)$ into itself. Thus, as in Jaśkiewicz [8], the definition of $P^{\prime}(\cdot \mid x)$ and assumptions 21] yield

$$
\left|T v_{1}(x)-T v_{2}(x)\right| \leq \sup _{\xi \in \Gamma(x) \cup\{x\}}\left|P^{\prime} v_{1}(\xi)-P^{\prime} v_{2}(\xi)\right|,
$$

which implies

$$
\left|T v_{1}(x)-T v_{2}(x)\right| \leq\left\|v_{1}-v_{2}\right\|_{W} \sup _{\xi \in \Gamma(x) \cup\{x\}} P^{\prime} W(\xi) \leq\left\|v_{1}-v_{2}\right\|_{W} \beta^{\prime} W(x)
$$

with $\beta^{\prime}$ as in Lemma 3.3 i.e., $T$ is a contraction in $C_{W}(E)$. Therefore, there is a fixed point $w_{0}$ in $C_{W}(E)$, i.e.,

$$
w_{0}(x)=\inf _{\xi \in \Gamma(x) \cup\{x\}}\left\{L(x, \xi)-g \tau(\xi)+P w_{0}(\xi)-\delta \gamma(\xi) \int_{D} w_{0}(z) m(\mathrm{~d} z)\right\} .
$$


Hence, the rest of the proof in Jaśkiewicz [8] shows that

$$
\int_{D} w_{0}(z) m(\mathrm{~d} z)=0
$$

and that $\left(g, w_{0}\right)$ is a solution to 29 . Moreover, we deduce

$$
\lim _{n \rightarrow \infty} \frac{\mathbb{E}_{x}^{\nu}\left\{w_{0}\left(X_{n}\right)\right\}}{n}=0,
$$

similarly to Luque-Vásquez and Hernández-Lerma [11, Lemma 4.3(c)].

5. Existence of an optimal control. To obtain an optimal control of the same type as in the [15] (i.e., based on the exit times of $\left\{x \in E: w_{0}(x)<M w_{0}(x)\right\}$ ), we will use the following additional assumption:

Knowing that the equation $h(x)=\ell(x)-j \tau(x)+P h(x)$, as a particular case of Theorem 4.1, has a solution $(j, h)$ in $\mathbb{R}^{+} \times C_{W}(E)$ and $j=\tilde{J}(x, 0,0)($ since $\Gamma(x)=\{x\}$ means 'no control', denoted by $\nu=0)$, we assume that

$$
h \text { is also bounded above, }
$$

see Remark 5.1 below for a discussion on this assumption.

Theorem 5.1. Under Assumption 2.1 and (15), (16), (18) and (22), which is also complemented with

$$
M w_{0} \text { is bounded below, }
$$

as well as (20), 21], (30), the constant $\mu_{0}$ obtained in Theorem 4.1 satisfies

$$
\mu_{0}=\inf \left\{\tilde{J}(x, 0, \nu): \nu \in \mathcal{V}_{0}\right\}
$$

and there exists an optimal feedback control based on the exit times of the continuation region $\left[w_{0}<M w_{0}\right]$.

Proof. First consider the case $\mu_{0}=j$. Exactly as in the proof of [15, inequality (5.6)], it is shown that $\mu_{0} \leq \tilde{J}(x, 0, \nu)$ for any $\nu$ in $\mathcal{V}_{0}$, i.e., $\mu_{0} \leq j$. Thus, if $\mu_{0}=j$ then $\mu_{0}=\inf \left\{\tilde{J}(x, 0, \nu): \nu \in \mathcal{V}_{0}\right\}=j=\tilde{J}(x, 0,0)$, and $\nu=0$ is optimal.

Now let us consider the case $\mu_{0}<j$. Note that assumption 30 implies that there exists a constant $K$ such that $h(x) \leq K$, for any $x$. Thus, we can define the function $\tilde{h}(x)=h(x)-K \leq 0$ to make the translation by $\tilde{h}$ in the equation of $w_{0}$. Indeed, since we have $\ell(x)=\tilde{h}(x)-P \tilde{h}(x)+j \tau(x)$, we obtain

$$
\tilde{w}(x)=\min \left\{\tilde{\psi},\left(j-\mu_{0}\right) \tau(x)+P \tilde{w}(x)\right\},
$$

with $\tilde{w}(x)=w_{0}(x)-\tilde{h}(x)$ and $\tilde{\psi}(x)=M w_{0}(x)-\tilde{h}(x)=M w_{0}(x)-h(x)+K$, and in view of (31), we can choose $K$ sufficiently large to have $\tilde{\psi} \geq 0$.

Hence, we have an equation corresponding to an (ergodic) stopping time with a strictly positive running cost and a positive stopping cost $\tilde{\psi}$, a priori unbounded.

In order to solve this problem as in [15. Theorem 5.1], for $\mu_{0}<j$, we need to extend the results of Bensoussan [1, Section 7.4, pp. 74-77] on discrete-time (ergodic) optimal stopping time as follows: if $\Phi$ is a continuous linear operator from $C_{W}(E)$ into itself, and

$$
\psi \in C_{W}(E), \quad \psi \geq 0, \quad \ell \in C_{b}(E), \quad \ell(x) \geq \ell_{0}>0
$$


then the equation

$$
u(x)=\min \{\psi(x), \ell(x)+\Phi u(x)\}
$$

has a unique positive solution in $C_{W}(E)$ and the optimal stopping time $\hat{\eta}$ satisfies $\mathbb{E}\{\hat{\eta}\} \leq$ $k W(x)$, with $k=\|\psi\|_{W} / \ell_{0}$.

We will skip the details, since this is a slight adaption of the bounded case.

Next, the remaining of the proof is similar to [15, Theorem 5.1], with the same optimal control $\hat{\eta}_{i+1}=\inf \left\{n \geq \hat{\eta}_{i}: w_{0}\left(X_{n}\right)=M w_{0}\left(X_{n}\right)\right\}$, where $\mathbb{E}_{x}\left\{\hat{\eta}_{i}\right\}<\infty$, for any $x$.

REMARK 5.1. If $f(x, y)=f(x)$ does not depend on $y$ and $x_{t}$ has a unique invariant probability measure $\zeta$, and the zero-potential

$$
x \mapsto h(x)=\mathbb{E}_{x}\left\{\int_{0}^{\infty}\left[f\left(x_{t}\right)-\bar{f}\right] \mathrm{d} s\right\}, \quad \text { with } \bar{f}=\zeta(f)>0,
$$

is continuous and satisfies

$$
\mathbb{E}_{x}\left\{h\left(x_{\theta}\right)\right\}=h(x)-\mathbb{E}_{x}\left\{\int_{0}^{\theta}\left[f\left(x_{t}\right)-\bar{f}\right] \mathrm{d} s\right\}, \quad \forall x,
$$

and for any bounded stopping time $\theta$, then $j=\bar{f}$ and the condition " $\{x: f(x) \geq \bar{f}\}$ is compact" ensures that (30) is satisfied (see Palczewski and Stettner [20]). If $f$ depends also on $y$ then one can find a similar condition with $\bar{f}=\zeta(f)$, where $\zeta(\mathrm{d} x, \mathrm{~d} y)$ is supposed to be a unique invariant probability measure of $\left(x_{t}, y_{t}\right)$ and there is a continuous zeropotential $h(x, y)$.

Now, we would like to obtain that $\mu_{0}$ is also the optimal cost given by $(13)$. To proceed along the lines of the case $E$ compact, we first obtain another form of the HJB equation for $w(x, y)$.

THEOREM 5.2. If the assumptions of Theorem 5.1 and the following condition on the weight function $W$ : there exists a positive constant $0<k<k_{0} \leq \lambda(x, y) \leq k_{1}$ such that

$$
\mathbb{E}_{x}\left\{\mathrm{e}^{-k t} W\left(x_{t}\right)\right\} \leq W(x), \quad \forall t \geq 0, \forall x \in E,
$$

are fulfilled, then the function $w(x, y)$ defined by (24) satisfies

$$
-A_{x y} w(x, y)+\lambda(x, y)[w(x, 0)-M w(x, 0)]^{+}=f(x, y)-\mu_{0} .
$$

Proof. Recall that $w(x, y)$ is defined by

$$
w(x, y)=\mathbb{E}_{x y}\left\{\int_{0}^{\tau}\left[f\left(x_{t}, y_{t}\right)-\mu_{0}\right] \mathrm{d} t+w_{0}\left(x_{\tau}\right)\right\}
$$

and use the precise space where $w(x, y)$ is actually defined. First, the inequalities

$$
\mathbb{E}_{x y}\left\{\int_{0}^{\tau}\left|f\left(x_{t}, y_{t}\right)-\mu_{0}\right| \mathrm{d} t\right\} \leq\left(\sup _{x, y}\left|f(x, y)-\mu_{0}\right|\right)\left(\sup _{x} \mathbb{E}_{x 0}\{\tau\}\right)
$$

and 10 show that the first term of $w(x, y)$ is bounded. Next, from the law of $\tau$, we have

$$
\mathbb{E}_{x y}\left\{w_{0}\left(x_{\tau}\right)\right\}=\mathbb{E}_{x}\left\{\int_{0}^{\infty} \lambda\left(x_{t}, y+t\right) \exp \left(-\int_{0}^{t} \lambda\left(x_{s}, y+s\right) \mathrm{d} s\right) w_{0}\left(x_{t}\right) \mathrm{d} t\right\} .
$$


Since $w_{0}$ belongs to $C_{W}(E)$ we have $\left|w_{0}\left(x_{t}\right)\right| \leq\left\|w_{0}\right\|_{W} W\left(x_{t}\right)$, and the bound on the density $\lambda$ yields

$\mathbb{E}_{x y}\left\{w_{0}\left(x_{\tau}\right)\right\} \leq \int_{0}^{\infty} k_{1} \mathrm{e}^{-k_{0} t} \mathbb{E}_{x}\left\{w_{0}\left(x_{t}\right)\right\} \mathrm{d} t \leq k_{1}\left\|w_{0}\right\|_{W} \int_{0}^{\infty} \mathrm{e}^{-\left(k_{0}-k\right) t} \mathbb{E}_{x}\left\{\mathrm{e}^{-k t} W\left(x_{t}\right)\right\} \mathrm{d} t$, and together with the hypothesis 32 , it follows that

$$
\left|\mathbb{E}_{x y}\left\{w_{0}\left(x_{\tau}\right)\right\}\right| \leq \frac{k_{1}}{k_{0}-k}\left\|w_{0}\right\|_{W} W(x), \quad \forall(x, y) \in E \times \mathbb{R}^{+} .
$$

This leads us to introduce $C_{W}\left(E \times \mathbb{R}^{+}\right)$, similarly to $C_{W}(E)$, as the space of all real-valued continuous functions $v$ on $E \times \mathbb{R}^{+}$such that

$$
\|v\|_{W}=\sup \left\{|v(x, y)| / W(x):(x, y) \in E \times \mathbb{R}^{+}\right\}<\infty,
$$

and to affirm that $w(x, y)$ belongs to $C_{W}\left(E \times \mathbb{R}^{+}\right)$.

Now, $w(x, y)$ can be written as

$$
\begin{aligned}
w(x, y)=\mathbb{E}_{x}\left\{\int_{0}^{\infty} \exp \left(-\int_{0}^{t} \lambda\left(x_{s}, y+s\right) \mathrm{d} s\right)\right. & \\
& \left.\times\left[f\left(x_{t}, y+t\right)-\mu_{0}+\lambda\left(x_{t}, y+t\right) w_{0}\left(x_{t}\right)\right] \mathrm{d} t\right\} .
\end{aligned}
$$

and the expression (see also Lemma 3.1

$$
\tilde{\Phi}(t) v(x, y)=\mathbb{E}_{x}\left\{\exp \left(-\int_{0}^{t} \lambda\left(x_{s}, y+s\right) \mathrm{d} s\right) v\left(x_{t}, y+t\right)\right\}
$$

defines a contraction semigroup on $C_{W}\left(E \times \mathbb{R}^{+}\right)$. Indeed, use assumption 32 to check the contraction property

$$
\begin{aligned}
|\tilde{\Phi}(t) v(x, y)| \leq \mathbb{E}_{x}\left\{\exp \left(-\int_{0}^{t} \lambda\left(x_{s}, y+s\right) \mathrm{d} s\right)\right. & \left.\left|v\left(x_{t}\right)\right|\right\} \\
& \leq \mathrm{e}^{-k_{0} t}\|v\|_{W} \mathbb{E}_{x}\left\{W\left(x_{t}\right)\right\} \leq\|v\|_{W} W(x) .
\end{aligned}
$$

Thus, if $R$ is the potential corresponding to $\{\tilde{\Phi}(t): t \geq 0\}$ then the function $w(x, y)$ becomes

$$
w(x, y)=R \varphi(x, y), \quad \text { with } \quad \varphi(x, y)=f(x, y)-\mu_{0}+\lambda(x, y) w_{0}(x),
$$

and again, if view of assumption (32), $R$ is a (linear) bounded operator on $C_{W}\left(E \times \mathbb{R}^{+}\right)$. Therefore, by means of Dynkin [5, Theorem 1.7', pp. 41-42], the function $w$ is the solution of the equation

$$
-\left(A_{x}+\partial_{y}\right) w+\lambda w=f-\mu_{0}+\lambda w_{0},
$$

where $A_{x}+\partial_{y}-\lambda$ is the weak generator of $\{\tilde{\Phi}(t): t \geq 0\}$.

Since [15, Lemma 5.6] is still valid under the current assumptions, we obtain

$$
w_{0}(x)=\min \{w(x, 0), M w(x, 0)\}=w(x, 0)-[w(x, 0)-M w(x, 0)]^{+} ;
$$

and rearranging the terms in the previous equation for $w(x, y)$, we deduce

$$
-A_{x y} w+\lambda[w(x, 0)-M w(x, 0)]^{+}=f-\mu_{0}
$$

as desired.

REMARK 5.2. As seen in the next section, condition 32 is not very restrictive. 
Lemma 5.1. Define $\mathcal{V}_{w^{+}} \subset \mathcal{V}$, the class of controls satisfying

$$
\frac{1}{T} \mathbb{E}_{x y}^{\nu}\left\{w^{+}\left(x_{T}, y_{T}\right)\right\} \rightarrow 0 \text { as } T \rightarrow \infty .
$$

Under the assumptions of Theorem 5.2 we have

$$
\mu_{0} \leq J(x, y, \nu), \quad \forall \nu \in \mathcal{V}_{w^{+}},
$$

provided $\mathcal{V}_{w^{+}} \neq \emptyset$.

Proof. Since assumption (32) yields, for every $t \geq 0, x, y$,

$$
\mathbb{E}_{x y}\left|w\left(x_{t}, y_{t}\right)\right| \leq\|w\|_{W} \mathbb{E}\left\{W\left(x_{t}\right)\right\} \leq \mathrm{e}^{k_{0} t} W(x)<\infty,
$$

the HJB equation 33 implies that

$$
M_{t}=\int_{0}^{t}\left[f\left(x_{s}, y_{s}\right)-\mu_{0}\right] \mathrm{d} s+w\left(x_{t}, y_{t}\right)
$$

is a $P_{x y}$-submartingale. As in [15, Theorem 5.8], sinc $e^{2}$

$$
w\left(x_{\theta_{i}}^{i-1}, 0\right) \leq c\left(x_{\theta_{i}}^{i-1}, \xi_{i}\right)+w\left(\xi_{i}, 0\right), \quad \forall i \geq 1,
$$

we deduce that

$$
w(x, y) \leq \mathbb{E}_{x y}^{\nu}\left\{\int_{0}^{T}\left[f\left(x_{t}, y_{t}\right)-\mu_{0}\right] \mathrm{d} t+\sum_{i=1}^{\infty} c\left(x_{\theta_{i}}^{i-1}, \xi_{i}\right) \mathbb{1}_{\theta_{i}<T}+w\left(x_{T}, y_{T}\right)\right\} .
$$

Hence, dividing by $T$ and letting $T \rightarrow \infty$ we deduce that $\mu_{0} \leq J(x, y, \nu)$, as long as $\nu$ belongs to $\mathcal{V}_{w^{+}}$.

Lemma 5.2. Define $\mathcal{V}_{w^{-}} \subset \mathcal{V}$ similarly to the class $\mathcal{V}_{w^{+}}$with $w^{-}$replacing $w^{+}$. Under the assumptions of Theorem 5.2 and $\hat{\nu} \in \mathcal{V}_{w^{-}}$we have $\mu_{0} \leq J(x, y, \hat{\nu})$.

Proof. Let $(\theta, \xi)$ be the first impulse of an arbitrary admissible impulse control $\nu$ in $\mathcal{V}$, and $T$ be a finite stopping time. We are going to show that

$$
\begin{aligned}
& \mathbb{E}_{x y}^{\nu}\left\{\int_{0}^{\theta}\left[f\left(x_{t}, y_{t}\right)-\mu_{0}\right] \mathrm{d} t+c\left(x_{\theta}^{0}, \xi\right)+w(\xi, 0)\right\} \\
& \geq \mathbb{E}_{x y}^{\nu}\left\{\int_{0}^{T \wedge \theta}\left[f\left(x_{t}, y_{t}\right)-\mu_{0}\right] \mathrm{d} t+\mathbb{1}_{\theta<T}\left[c\left(x_{\theta}^{0}, \xi\right)+w(\xi, 0)\right]+\mathbb{1}_{\theta \geq T} w\left(x_{T}, y_{T}\right)\right\} .
\end{aligned}
$$

Indeed, it was seen that $M_{t}$ is a $P_{x y}$ submartingale, and thus $E_{x y}\left\{M_{\theta} \mid \mathcal{F}_{T \wedge \theta}\right\} \geq M_{T \wedge \theta}$, i.e.,

$\mathbb{E}_{x y}^{\nu}\left\{\int_{0}^{\theta}\left[f\left(x_{t}, y_{t}\right)-\mu_{0}\right] \mathrm{d} t+w\left(x_{\theta}^{0}, y_{\theta}\right) \mid F_{T \wedge \theta}\right\} \geq \int_{0}^{T \wedge \theta}\left[f\left(x_{t}, y_{t}\right)-\mu_{0}\right] \mathrm{d} t+w\left(x_{T \wedge \theta}^{0}, y_{T \wedge \theta}\right)$.

This yields

$$
\begin{aligned}
\mathbb{E}_{x y}^{\nu}\left\{\int_{0}^{\theta}\left[f\left(x_{t}, y_{t}\right)-\mu_{0}\right] \mathrm{d} t+\mathbb{1}_{\theta \geq T} w\left(x_{T}^{0}, y_{T}\right)+\mathbb{1}_{\theta<T} w\left(x_{\theta}^{0}, y_{\theta}\right) \mid \mathcal{F}_{T \wedge \theta}\right\} \\
\geq \int_{0}^{T \wedge \theta}\left[f\left(x_{t}, y_{t}\right)-\mu_{0}\right] \mathrm{d} t+\mathbb{1}_{\theta \geq T} w\left(x_{T}^{0}, y_{T}\right)+\mathbb{1}_{\theta<T} w\left(x_{\theta}^{0}, y_{\theta}\right),
\end{aligned}
$$

\footnotetext{
${ }^{2}$ this inequality holds at impulse times, even if in general we do not have $w \leq M w$.
} 
and, after remarking that $\mathbb{1}_{\theta<T} w\left(x_{\theta}^{0}, y_{\theta}\right)$ is $\mathcal{F}_{T \wedge \theta}$ measurable, adding $\mathbb{1}_{\theta<T}\left[c\left(x_{\theta}^{0}, y_{\theta}\right)+\right.$ $w(\xi, 0)$ ] on both sides, and taking expectation, we have

$$
\begin{aligned}
& \mathbb{E}_{x y}^{\nu}\left\{\int_{0}^{\theta}\left[f\left(x_{t}, y_{t}\right)-\mu_{0}\right] \mathrm{d} t+\mathbb{1}_{\theta \geq T} w\left(x_{T}^{0}, y_{T}\right)+\mathbb{1}_{\theta<T}\left[c\left(x_{\theta}^{0}, y_{\theta}\right)+w(\xi, 0)\right]\right\} \\
& \quad \geq \mathbb{E}_{x y}^{\nu}\left\{\int_{0}^{T \wedge \theta}\left[f\left(x_{t}, y_{t}\right)-\mu_{0}\right] \mathrm{d} t+\mathbb{1}_{\theta \geq T} w\left(x_{T}^{0}, y_{T}\right)+\mathbb{1}_{\theta<T}\left[c\left(x_{\theta}^{0}, y_{\theta}\right)+w(\xi, 0)\right]\right\} .
\end{aligned}
$$

Now, we have seen that, at impulse times,

$$
w\left(x_{\theta}^{0}, y_{\theta}\right) \leq c\left(x_{\theta}^{0}, \xi\right)+w(\xi, 0)
$$

and hence, using this on the left hand side, we obtain (34).

Let us verify that if $\hat{\nu}$ belongs to $\mathcal{V}_{w^{-}}$(which is defined similarly to $\mathcal{V}_{w^{+}}$), then $\mu_{0} \geq J(\hat{\nu})$.

Indeed, from the explicit optimal stopping time representation (as in [15])

$$
w(x, y)=\inf _{\theta} \mathbb{E}_{x y}\left\{\int_{0}^{\theta}\left[f\left(x_{t}, y_{t}\right)-\mu_{0}\right] \mathrm{d} t+M w\left(x_{\theta}, 0\right)\right\},
$$

for any admissible stopping time $\theta$, and using the optimal stopping time $\hat{\theta}_{1}$ and optimal impulse $\hat{\xi}_{1}$ (corresponding to a minimizer of $\left.M w\right)$, we obtain

$$
\begin{aligned}
& w(x, y) \geq \mathbb{E}_{x y}^{\hat{\nu}}\left\{\int_{0}^{T \wedge \hat{\theta}_{1}}\left[f\left(x_{t}, y_{t}\right)-\mu_{0}\right] \mathrm{d} t+\mathbb{1}_{\hat{\theta}_{1}<T}\left[c\left(x_{\hat{\theta}_{1}}^{0}, \hat{\xi}_{1}\right)+w\left(\hat{\xi}_{1}, 0\right)\right]\right. \\
&\left.+\mathbb{1}_{\hat{\theta}_{1} \geq T} w\left(x_{T}, y_{T}\right)\right\} .
\end{aligned}
$$

By iterating this argument with $T$ constant, it follows that

$$
\begin{aligned}
w(x, 0)+\mu_{0} T \geq \mathbb{E}_{x 0}^{\hat{\nu}}\left\{\int_{0}^{T \wedge \hat{\theta}_{n}} f\left(x_{t}, y_{t}\right) \mathrm{d} t+\sum_{i=1}^{n-1} \mathbb{1}_{\hat{\theta}_{i}<T}\left[c\left(x_{\hat{\theta}_{i}}^{i-1}, \hat{\xi}_{i}\right)\right]\right. & \\
& \left.+\mathbb{1}_{\hat{\theta}_{i} \geq T}\left[w\left(x_{T \wedge \hat{\theta}_{n}}, y_{T \wedge \hat{\theta}_{n}}\right)\right]\right\} .
\end{aligned}
$$

Dividing by $T$, letting $T \rightarrow \infty$ and using $w=w^{+}-w^{-}$, the previous inequality yields $\mu_{0} \geq J(\hat{\nu})$ as desired.

THEOREM 5.3. Under the assumptions as in Theorem 5.2, and assuming that $\hat{\nu}$ belongs to $\mathcal{V}_{w^{+}} \cap \mathcal{V}_{w^{-}}$, i.e.,

$$
\frac{1}{T} \mathbb{E}_{x y}^{\nu}\left\{\left|w\left(x_{T}, y_{T}\right)\right|\right\} \rightarrow 0 \text { as } T \rightarrow \infty
$$

we have

$$
\mu_{0}=\inf \left\{J(x, y, \nu): \nu \in \mathcal{V}_{w^{+}}\right\}=J(x, y, \hat{\nu}),
$$

where the constant $\mu_{0}$ and the optimal feedback control $\hat{\nu}$ are as in Theorem 5.1, translated by $\tau$, see (7).

Proof. From Lemmas 5.1 and $5.2, \mu_{0} \leq J(\nu)$, for every $\nu \in \mathcal{V}_{w^{+}}$and $\mu_{0} \geq J(\hat{\nu})$, and since $\hat{\nu} \in \mathcal{V}_{w^{+}}$, we deduce 36 .

REMARK 5.3.

(a) If $w$ is bounded then, obviously, $\mathcal{V}_{w+}=\mathcal{V}_{w^{-}}=\mathcal{V}$ and assumption 35 of Theorem 5.3 is satisfied. 
(b) Also, based on the inequality

$$
\left|\mathbb{E}_{x y}^{\nu}\left\{w\left(x_{T}, y_{T}\right)\right\}\right| \leq\|w\|_{W} \mathbb{E}_{x}^{\nu}\left\{W\left(x_{T}\right)\right\},
$$

if we assume that

$$
A_{x} W(x) \leq-\beta W(x)+b, \quad \beta>0,
$$

then one can show that for $\nu=0$ (no control), we have

$$
\lim _{T \rightarrow \infty} \frac{1}{T} \mathbb{E}_{x}^{0}\left\{W\left(x_{T}\right)\right\}=0,
$$

i.e., $\nu=0$ belongs to $\mathcal{V}_{w+}$ and $\mathcal{V}_{w^{-}}$, which are therefore nonempty subset of admissible impulse controls.

Now, let us mention some cases (examples and comments) where our assumptions are satisfied.

6. About the ergodicity condition. If we assume that there exists a norm-like (or Lyapunov type) function $V(x) \geq 1$, for every $x$ in $E$ (i.e., also $V(x) \rightarrow \infty$ as $|x| \rightarrow \infty$, and in the domain of the extended generator) and constant $\beta>0$ and $\gamma \geq 0$ such that $A_{x} V(x) \leq-\beta V(x)+\gamma$, where $A$ is the infinitesimal generator of $x_{t}$; then $P V(x) \leq$ $\beta^{\prime} V(x)+\gamma^{\prime}$ for some constants $0<\beta^{\prime}<1$ and $\gamma^{\prime} \geq 0$, where $P$ is the operator given by (27). Indeed, to check this assertion, use Dynkin's formula and the assumptions on $V$ to get

$$
P V(x)=\mathbb{E}_{x 0}\left\{V\left(x_{\tau}\right)\right\} \leq V(x)-\beta \mathbb{E}_{x 0}\left\{\int_{0}^{\tau} V\left(x_{t}\right) \mathrm{d} t\right\}+\gamma \mathbb{E}_{x 0}\{\tau\} .
$$

Moreover, one checks that

$$
\begin{aligned}
\mathbb{E}_{x 0}\left\{\int_{0}^{\tau} V\left(x_{t}\right) \mathrm{d} t\right\}= & \mathbb{E}_{x}\left\{\int_{0}^{\infty} \exp \left(-\int_{0}^{t} \lambda\left(x_{s}, s\right) \mathrm{d} s\right) V\left(x_{t}\right) \mathrm{d} t\right\} \\
& \geq \varepsilon \mathbb{E}_{x}\left\{\int_{0}^{\infty} \exp \left(-\int_{0}^{t} \lambda\left(x_{s}, s\right) \mathrm{d} s\right) V\left(x_{t}\right) \lambda\left(x_{t}, t\right) \mathrm{d} t\right\}=\varepsilon P V(x),
\end{aligned}
$$

with $\varepsilon=1 / \sup \{\lambda(x, y): x, y\}$ (i.e., $\left.\geq 1 / k_{1}\right)$. Hence $P V(x) \leq V(x)-\beta \varepsilon P V(x)+\gamma^{\prime}$, with $\gamma^{\prime}=\gamma \sup _{x \in E} \mathbb{E}_{x 0}\{\tau\}=\gamma a_{2}$ (see Remark 2.3) and finally the desired inequality with $\beta^{\prime}=1 /(1+\beta \varepsilon)$ follows.

Now, for instance, if for any compact set $C \subset E$ there exist a constant $0<\alpha<1$ and a probability $\nu$ such that

$$
P \mathbb{1}_{B}(x) \geq \alpha \mathbb{1}_{C}(x) \nu(B), \quad \forall B \in \mathcal{B}(E),
$$

then the condition $P V(x) \leq \beta^{\prime} V(x)+\gamma^{\prime}$ for every $x$ in $E$ is equivalent to $P V(x) \leq$ $\beta_{1} V(x)+\beta_{2} \mathbb{1}_{C}(x)$ for every $x$ in $E$, for some constants $0<\beta_{1}<1$ and $\beta_{2} \geq 0$ and a compact $C$ (this is a particular case of Meyn and Tweedie [18, Lemma 15.2.8, pp. 379-380]).

Another point is that under the same assumption $A_{x} V(x) \leq-\beta V(x)+\gamma$ for every $x$ in $E$, we deduce that for any constant $k>0$ the function $V_{k}(x)=V(x)+\gamma / k$ satisfies $A_{x} V_{k}(x) \leq k V_{k}(x)$ for every $x$ in $E$. (Indeed, $A_{x} V_{k}(x)=A_{x} V(x) \leq k V(x)+\gamma=k V_{k}(x)$ ) Since $V_{k} \geq V \geq 1$ we have $\mathbb{E}_{x}\left\{\mathrm{e}^{-k t} V_{k}\left(x_{t}\right)\right\} \leq V_{k}(x)$ for every $x$ in $E$ and $t \geq 0$. This means that the condition $A_{x} V(x) \leq-\beta V(x)+\gamma$ for every $x$ in $E$ implies that the property 32 on the weight function $W$ required in Theorem 5.1 is satisfied. 
REMARK 6.1. Note that in Meyn and Tweedie [17, Theorem 6.1, pp. 536-537] it is proved that the condition $A_{x} V(x) \leq-\beta V(x)+\gamma$ for every $x$ in $E$ implies (in particular) the exponential ergodicity of $x_{t}$. It should be observed that this condition is to be written with truncation of the process $x_{t}$, i.e., first get an increasing sequence $\left\{\mathcal{O}_{n}\right\}$ of open sets with compact closure and such that $\mathcal{O}_{n} \uparrow E$, and then consider the first entrance time $T_{n}$ of $E \backslash \mathcal{O}_{n}$. Thus, define $x_{t}^{n}=x_{t}$ for any $t<T_{n}$ and $x_{t}^{n}=x_{n}^{*}$ for any $t \geq T_{n}$, with a fixed state $x_{n}^{*}$ in $E \backslash \mathcal{O}_{n}$ (e.g., $x_{n}^{*}=x_{T_{n}}^{n}$ ) and $A_{x}^{n}$ the extended generator of $x_{t}^{n}$, so that the condition becomes $A_{x}^{n} V(x) \leq-\beta V(x)+\gamma$ for every $x$ in $\mathcal{O}_{n}$.

Proposition 6.1. If, besides the assumptions of Theorem 5.1, we assume also that

(i) $\Gamma(x) \subset K$, for every $x$ and some fixed compact set $K$;

(ii) for some $Y>0$ the stopping time $T_{K Y}=\inf \left\{t>0:\left(x_{t}, y_{t}\right) \in K \times[0, Y]\right\}$ satisfies $E\left\{T_{K Y}\right\}<\infty$, for every $x, y$;

(iii) $L=\left\{(x, y): f(x, y) \leq \mu_{0}\right\}$ is compact and $T_{L}=\inf \left\{t>0:\left(x_{t}, y_{t}\right) \in L\right\}$ satisfies $\mathbb{E}_{x y}\left\{T_{L}\right\}<\infty$, for every $x, y$;

then the function $w$ is bounded and therefore the conditions of Theorem 5.3 are satisfied (actually, $\mathcal{V}_{w+}=\mathcal{V}_{w^{-}}=\mathcal{V}$ ).

Proof. First we show that $w$ is bounded above and next below. Indeed, for any function $v$ locally bounded in $E$ (in particular continuous), assumption 15 on the switching cost $c$ yields

$$
c_{0}+\inf _{\xi \in K} v(\xi) \leq M v(x) \leq\|c\|+\sup _{\xi \in K} v(\xi),
$$

and therefore, $w_{0} \leq M w_{0}$ implies $w_{0} \leq\|c\|+\sup _{\xi \in K} w_{0}(\xi)=b_{1}$. Hence, from

$$
w(x, y)=\mathbb{E}_{x y}\left\{\int_{0}^{\tau_{1}}\left[f\left(x_{s}, y_{s}\right)-\mu_{0}\right] \mathrm{d} s+w_{0}\left(x_{\tau_{1}}\right)\right\}
$$

it follows that $w(x, y)=\varphi(x, y)+\mathbb{E}_{x y}\left\{w_{0}\left(x_{\tau_{1}}\right)\right\}$ with $\varphi$ bounded, i.e., $w^{+}(x, y) \leq\|\varphi\|+b_{1}$, and $b_{1}$ can be assumed nonnegative. Thus $w^{+}$is bounded.

Next, using inequality (34) with $T=T_{K Y} \wedge T_{L}$, and observing that $f-\mu_{0} \geq 0$ for any $0 \leq t \leq T$, we have

$$
\begin{aligned}
& w(x, y) \geq \mathbb{E}_{x y}^{\nu}\left\{\mathbb { 1 } _ { \theta < T } \left[c\left(x_{\theta}^{0}, \xi\right)+\right.\right.\left.w(\xi, 0)]+\mathbb{1}_{\theta \geq T} w\left(x_{T}, y_{T}\right)\right\} \\
& \geq \mathbb{E}_{x y}\left\{\mathbb{1}_{\theta<T}\right\}\left[\inf _{x, \xi} c(x, \xi)+\inf _{\xi \in K} w(\xi, 0)\right] \\
& \quad+\mathbb{E}_{x y}\left\{\mathbb{1}_{\theta \geq T}\right\} \inf \{w(x, y):(x, y) \in(K \times[0, Y]) \cup L\},
\end{aligned}
$$

i.e., $w(x, y)$ is necessarily bounded below.

7. Diffusion processes. Consider a diffusion in $E=\mathbb{R}^{d}$ given by $\mathrm{d} x_{t}=b\left(x_{t}\right)+$ $\sigma\left(x_{t}\right) \mathrm{d} B_{t}$ with $b$ and $\sigma$ are continuously differentiable functions, $b$ having linear growth, $\sigma$ being bounded, and $\sigma \sigma^{*}$ is strictly elliptic (uniformly in $x$ ). Classic results on the diffusion processes (e.g., Friedman [7, Sections 1.6 and 2.4, pp. 22-25 and pp. 42-48], Ladyženskaja et al. [9, Section IV.11, pp. 356-364], and references therein) show that $x_{t}$ admits a transition density function $p\left(x, t, x^{\prime}\right)$, which is strictly positive and continuous in $x, x^{\prime}$. As a consequence, given any $t^{*}>0$ and any compact $C \subset \mathbb{R}^{d}$ there exist a constant 
$0<\alpha<1$ and a probability $\nu$ such that

$$
P\left(x, t^{*}, B\right)=\int_{B} p\left(x, t^{*}, x^{\prime}\right) \mathrm{d} x^{\prime} \geq \alpha \mathbb{1}_{C}(x) \nu(B), \quad \forall B \in \mathcal{B}\left(\mathbb{R}^{d}\right), \forall x \in \mathbb{R}^{d} .
$$

Equivalently, for any $k>0$, a similar property holds for the resolvent chain corresponding to the kernel

$$
R_{k}(x, B)=\int_{0}^{\infty} k \mathrm{e}^{-k t}\left(\Phi(t) \mathbb{1}_{B}\right)(x) \mathrm{d} t,
$$

i.e., for any compact $C \subset \mathbb{R}^{d}$ there exist a constant $0<\alpha<1$ and a probability $\nu$ such that

$$
R_{k}(x, B) \geq \alpha \mathbb{1}_{C}(x) \nu(B), \quad \forall B \in \mathcal{B}\left(\mathbb{R}^{d}\right), \forall x \in \mathbb{R}^{d} .
$$

As a consequence, since

$$
\begin{aligned}
P(x, B)=\mathbb{E}_{x}\left\{\int_{0}^{\infty} \exp \left(-\int_{0}^{t} \lambda\left(x_{s}, s\right) \mathrm{d} s\right) \lambda\left(x_{t}, t\right) \mathbb{1}_{B}\left(x_{t}\right) \mathrm{d} t\right\} & \\
& \geq \frac{k_{0}}{k_{1}} R_{k_{1}}(x, B) \geq \frac{k_{0}}{k_{1}} \alpha \mathbb{1}_{C}(x) \nu(B),
\end{aligned}
$$

we deduce that

$$
P(x, B) \geq \beta_{0} \mathbb{1}_{C}(x) \nu(B), \quad \forall B \in \mathcal{B}\left(\mathbb{R}^{d}\right), \forall x \in \mathbb{R}^{d},
$$

as assumed in 21].

Now in view of Section 6 we see that if there exists a Lyapunov function such that $A_{x} V(x) \leq-\beta V(x)+\gamma$ for every $x$ in $\mathbb{R}^{d}$ then the whole hypothesis 21 is satisfied as well as the additional condition of Theorem 5.1. Also, the Markov process $x_{t}$ is exponentially ergodic and one can show that when the function $f(x, y)=f(x)$ depend only on $x$, there exists a function $h(x)$ satisfying the additional assumption of Section 5

Finally, if we are in the case of $\Gamma(x) \subset K_{0}$, a fixed compact, for every $x$ in $\mathbb{R}^{d}$, then that all the results of Section 5 are valid. Moreover, as a simple example with the Lyapunov function $V(x)=x^{2}+1$ is the diffusion $x_{t}$ in $\mathbb{R}$ defined by $\mathrm{d} x_{t}=-x_{t} \mathrm{~d} t+\sigma\left(x_{t}\right) \mathrm{d} B_{t}$, with $\sigma$ continuous and bounded, and such that $\sigma^{2}(x) \geq \alpha_{0}>0$. Certainly, more complicate examples can be found in Meyn and Tweedie [17, Section 8, pp. 537-539].

Some classes of diffusion processes with jumps give examples satisfying our ergodic assumptions, e.g., Masuda [12].

\section{References}

[1] A. Bensoussan, Dynamic Programming and Inventory Control, Stud. Probab. Optim. Statist., IOS Press, Amsterdam, 2011.

[2] A. Bensoussan, J.-L. Lions, Applications des inéquations variationnelles en contrôle stochastique, Méthodes Math. Inform. 6, Dunod, Paris, 1978.

[3] A. Bensoussan, J.-L. Lions, Contrôle impulsionnel et inéquations quasi-variationnelles, Méthodes Math. Inform. 11, Gauthier-Villars, Paris, 1982.

[4] M. H. A. Davis, Markov Models and Optimization, Monogr. Statist. Appl. Probab. 49, Chapman \& Hall, London, 1993.

[5] E. B. Dynkin, Markov Processes, vol. 1, Grundlehren Math. Wiss. 121, Springer, Berlin, 1965. 
[6] S. N. Ethier, T. G. Kurtz, Markov Processes, Characterization and Convergence, Wiley Ser. Probab. Math. Statist. Wiley, New York, 1986.

[7] A. Friedman, Partial Differential Equations of Parabolic Type, Prentice-Hall, Englewood Cliffs, NJ, 1964.

[8] A. Jaśkiewicz, A fixed point approach to solve the average cost optimality equation for semi-Markov decision processes with Feller transition probabilities, Comm. Statist. Theory Methods 36 (2007), 2559-2575.

[9] O. A. Ladyženskaja, V. A. Solonnikov, N. N. Ural'ceva, Linear and quasilinear equations of parabolic type, Transl. Math. Monogr. 23, Amer. Math. Soc., Providence, RI, 1967.

[10] J. P. Lepeltier, B. Marchal, Théorie générale du contrôle impulsionnel markovien, SIAM J. Control Optim. 22 (1984), 645-665.

[11] F. Luque-Vásquez, O. Hernández-Lerma, Semi-Markov control models with average costs, Appl. Math. (Warsaw) 26 (1999), 315-331.

[12] M. Masuda, Ergodicity and exponential $\beta$-mixing bounds for multidimensional diffusions with jumps, Stochastic Process. Appl. 117 (2007), 35-56; Erratum: ibid. 119 (2009), 676678.

[13] J. L. Menaldi, M. Robin, On some optimal stopping problems with constraint, SIAM J. Control Optim. 54 (2016), 2650-2671.

[14] J. L. Menaldi, M. Robin, On some impulse control problems with constraint, SIAM J. Control Optim. 55 (2017), 3204-3225.

[15] J. L. Menaldi, M. Robin, On some ergodic impulse control problems with constraint, SIAM J. Control Optim. 56 (2018), 2690-2711.

[16] J. L. Menaldi, M. Robin, On optimal stopping and impulse control with constraint, in: Modeling, Stochastic Control, Optimization, and Applications IMA Vol. Math. Appl. 164, Springer, Cham, 2019, 427-450.

[17] S. P. Meyn, R. L. Tweedie, Stability of Markovian processes. III. Foster-Lyapunov criteria for continuous-time processes, Adv. in Appl. Probab. 25 (1993), 518-548.

[18] S. P. Meyn, R. L. Tweedie, Markov chains and stochastic stability, second ed., Cambridge Univ. Press, Cambridge, 2009.

[19] J. Palczewski, Ł. Stettner, Finite horizon optimal stopping of time-discontinuous functionals with applications to impulse control with delay, SIAM J. Control Optim. 48 (2010), 48744909.

[20] J. Palczewski, Ł. Stettner, Infinite horizon stopping problems with (nearly) total reward criteria, Stochastic Process. Appl. 124 (2014), 3887-3920.

[21] J. Palczewski, Ł. Stettner, Impulse control maximizing average cost per unit time: A nonuniformly ergodic case, SIAM J. Control Optim. 55 (2017), 936-960.

[22] M. Robin, Contrôle impulsionnel des processus de Markov, Thèse d'état, 1978, https://hal.archives-ouvertes.fr/tel-00735779/document.

[23] Ł. Stettner, Discrete time adaptive impulsive control theory, Stochastic Process. Appl. 23 (1986), 177-197.

[24] Ł. Stettner, On ergodic impulsive control problems, Stochastics 18 (1986), 49-72. 